\title{
A study to evaluate the clinical outcome of drug-eluting vs. bare-metal stents for acute coronary syndrome patients during commercial use in real setting
}

\author{
Chetan R. Patel ${ }^{\mathrm{a} * *}$, Mandev B. Patel ${ }^{\mathrm{b}}$
}

${ }^{a}$ Department of Pharmacology, Singhania University, Pacheri bari, Jhunjhunu, Rajasthan, India,

${ }^{\mathrm{b}}$ Department of Pharmacology, K.B. Raval College of Pharmacy, Gandhinagar, Gujarat, India

Received: 29 July 2012

Revised: 3 August 2012

Accepted: 14 August 2012

*Correspondence to:

Chetan R. Patel,

Email: chetanniper@gmail.com

\begin{abstract}
Background: This study compared clinical outcome of Drug Eluting Stents (DES) versus Bare Metal Stents (BMS) in coronary arteries in patients with Acute Coronary Syndromes.

Methods: A retrospective, observational study was carried out in an inpatient setting of the private tertiary care hospital. Patients with $>18$ years, diagnosed for Acute Coronary Syndromes (ACS), required intervention in coronary artery with implantation of Drug Eluting Stents (DES) or Bare Metal Stents (BMS) were recruited in the study. The data had been collected from file or database of the hospital. All subjects were followed for major adverse cardiac event.

Results: A total of 202 patients who underwent percutaneous coronary intervention (PCI) were enrolled into DES group $(n=101)$ and BMS group $(n=101)$. All patients were followed up at 1 month, 3 months, 6 months \& 12 months for Major Adverse Cardiac Events (MACE). Clinical outcomes during 12 months were compared between DES group \& BMS group. Overall MACE rates were reported non-significantly high in BMS group patients $(14.85 \%)$ compare to DES group patients $(8.91 \%)(\mathrm{p}=0.458)$. However, DES group had lower rates of death $(0.99 \%$ vs $1.98 \%, \mathrm{p}=0.57)$, rate of MI $(3.96 \%$ vs $4.95 \%$ $\mathrm{p}=0.73)$, rate of revascularization $(1.98 \%$ vs $3.96 \% \mathrm{p}=0.42) \&$ rate of sub acute thrombosis $(1.98 \%$ vs $3.96 \% \mathrm{p}=0.42)$ and higher rate of bleeding $(1.98 \%$ vs $0.99 \% \mathrm{p}=0.57$ ) compare to cohort-II.

Conclusions: The use of DES in the setting of Acute Coronary Syndrome is associated with lower Major Adverse Cardiac Event (MACE) rate compared to BMS without compromising the overall safety over the course of one-year follow-up. The long-term safety of drug-eluting stents needs to be ascertained in large, randomized trials.
\end{abstract}

Keywords: Drug Eluting Stent (DES), Bare Metal Stent (BMS), Major Adverse Cardiac Event (MACE), Acute Coronary Syndrome (ACS)

\section{INTRODUCTION}

Cardiovascular disease (CVD) is the leading cause of death and disability in the world. ${ }^{1}$ Among these, coronary artery disease (CAD) is the most prevalent manifestation and is associated with high mortality and morbidity. India has the highest burden of acute coronary syndromes in the world, yet little is known about the treatments and outcomes of these diseases. There is a need to document the characteristics, treatments, and outcomes of patients with acute coronary syndromes who were admitted to hospitals in India. ${ }^{2}$
Prospective, randomized clinical trials have shown that in-stent restenosis is reduced by the use of drug-eluting stents, as compared with bare-metal stents. However, the use of drug-eluting stents has rapidly been expanded to all types of patients, including those with more complicated coronary lesions and in acute settings. Recently metaanalyses of randomized trials ${ }^{3-4}$ and registries ${ }^{5}$ have raised concern about incomplete neointimal coverage with a subsequent increase in late stent thrombosis in patients with drug-eluting stents. ${ }^{6-7}$ One randomized trial indicated that the implantation of drug-eluting stents was associated with an early reduction in death and myocardial 
infarction-an improvement that was lost during the subsequent 6 to 18 months by a late increase in the same events. ${ }^{8}$ We determined that the evaluation of large clinical registries might provide useful information concerning the long-term efficacy and safety of drugeluting stents. Therefore, we evaluated the long-term outcome in all patients who underwent stent implantation.

\section{METHODS}

A retrospective, open label, observational study carried out in an inpatient setting of the private tertiary care hospital. Patients with $>18$ years, diagnosed for Acute Coronary Syndromes (ACS), required intervention in coronary artery with implantation of Drug Eluting Stents (DES) or Bare Metal Stents (BMS) were recruited in the study. The data had been collected from file or database of the hospital. All subjects were followed for major adverse cardiac event (MACE) including death, Myocardial infarction, Urgent revascularization, sub acute thrombosis \& bleeding at 1 month, 3 months, 6 months \& 12 months after PCI. All collected data was analyzed in its group for clinical outcomes. All variables were analyzed using percentage, mean \& standard deviation. Statistical difference between both cohorts was calculated by applying independent t-test $\&$ odds ratio.

\section{RESULTS}

There were 202 patients who underwent PCI between December 2008 and July 2009 was enrolled in retrospective, observational study. All these subjects were divided in two Cohorts:

Cohort-I: Patients implanted Drug Eluting Stent (DES) $(n=101)$ and

Cohort-II: Patients implanted Bare Metal stent (BMS) $(n=101)$

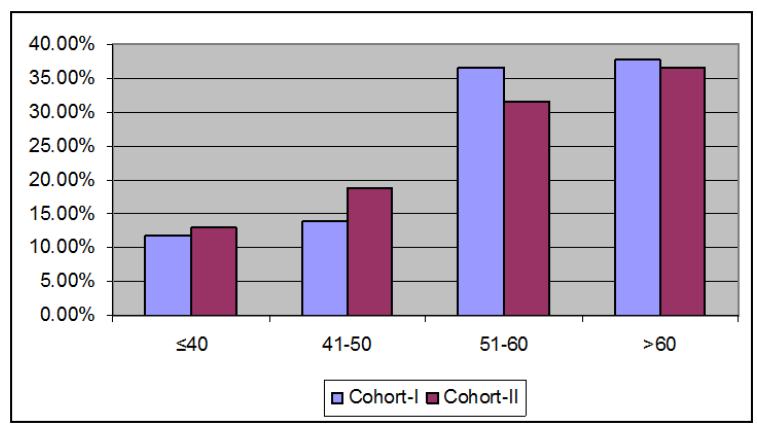

Figure 1: Age wise distribution of patients.

Out of the 202 patients, $87.13 \% \quad(n=88)$ and $89.11 \%$ $(n=90)$ were male patients in Cohort-I \& Cohort-II respectively. The average age of the patients were 56.34 \& 55.46 in Cohort-I \& Cohort-II respectively (Table 1). Risk of acute coronary syndrome was increased with increasing age (Figure 1). Average BMI in cohort I \& cohort II were $25.25 \mathrm{Kg} / \mathrm{m}^{2} \& 28.87 \mathrm{Kg} / \mathrm{m}^{2}$ respectively.
There was no significant difference in baseline demographic parameters between both groups (Table 1).

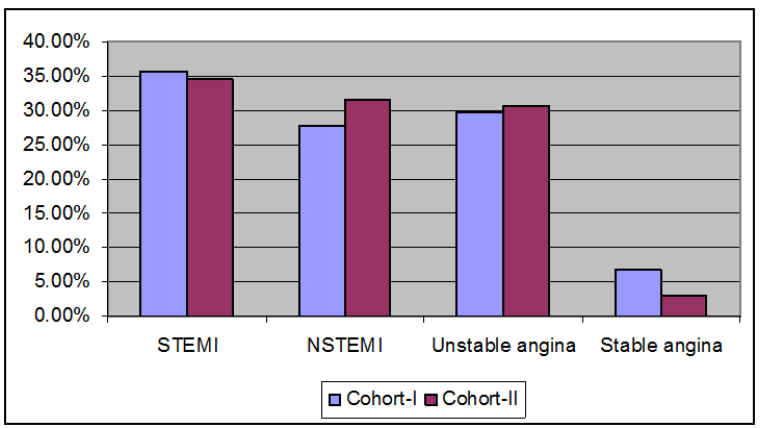

Figure 2: Diagnosis of patients.

There were high number of subjects were reported as STEMI in both groups. STEMI was reported $35.64 \%$ (36) Vs $34.66 \%$ (35), $\mathrm{p}=0.88$ in Cohort-I \& Cohort-II respectively. NSTEMI was reported $27.73 \%$ (28) Vs $31.68 \%$ (32), $\mathrm{p}=0.54$ in Cohort-I \& Cohort-II respectively. Unstable angina was reported $29.7 \%$ (30) Vs $30.69 \%$ (31), $\mathrm{p}=0.88$ in Cohort-I \& Cohort-II respectively. Stable angina was reported $6.93 \%$ (7) Vs $2.97 \%$ (3), $\mathrm{p}=0.21$ in Cohort-I \& Cohort-II respectively (Figure 2).

During coronary angiography (CAG), It was found that the main culprit vessel in coronary artery disease was LAD $(66.34 \%$ \& $54.45 \%$ in Cohort-I \& Cohort-II respectively) followed by RCA $(21.78 \%$ \& $23.76 \%$ in Cohort-I \& Cohort-II respectively). There were high numbers of subjects having coronary stenosis $>90 \%$ in both cohorts $(49.5 \%$ \& $52.47 \%$ in Cohort-I \& Cohort-II respectively). Detail coronary angiographic findings were shown in Table 2.

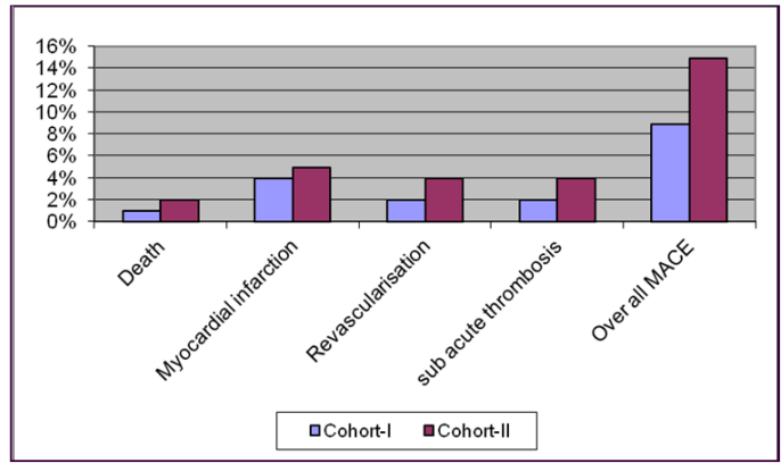

Figure 3: Major adverse cardiac events.

Overall MACE rates were reported non-significantly high in Cohort-I (8.91\%) compare to Cohort-II (14.85\%) ( $\mathrm{p}=$ 0.458). However, Cohort-I had lower rates of death (0.99\% vs $1.98 \%, \mathrm{p}=0.57)$, rate of MI $(3.96 \%$ vs $4.95 \%$ $\mathrm{p}=0.73)$, rate of revascularization $(1.98 \%$ vs $3.96 \%$ $\mathrm{p}=0.42) \&$ rate of sub acute thrombosis $(1.98 \%$ vs $3.96 \%$ $\mathrm{p}=0.42)$ and higher rate of bleeding $(1.98 \%$ vs $0.99 \%$ $\mathrm{p}=0.57$ ) compare to cohort-II (Figure 3). 
Over all MACE rate reported for both of the groups was $0.99 \%$ Vs $0.99 \%(\mathrm{p}=1)$ at one month, $0.99 \%$ Vs $2.97 \%$ $(\mathrm{p}=0.34)$ at three months, $2.97 \%$ Vs $3.96 \%(\mathrm{p}=0.7)$ at six months and $3.96 \%$ Vs $6.93 \%(\mathrm{p}=0.36)$ at twelve months in Cohort-I \& Cohort-II respectively (Figure 4).

Table 1: Demographic parameters.

\begin{tabular}{|llll|}
\hline Parameters & Cohort-I & Cohort-II & $p$-value \\
Age & $56.34 \pm 10.93$ & $55.46 \pm 12.47$ & 0.59 \\
Gender-Male & $87.13 \%(88)$ & $89.11 \%(90)$ & 0.66 \\
Weight & $68.02 \pm 11.33$ & $69.8 \pm 0.29$ & 0.29 \\
Height & $164.2 \pm 9.55$ & $164 \pm 7.08$ & 0.88 \\
BMI & $25.25 \pm 3.81$ & $28.87 \pm 3.74$ & 0.24 \\
BSA & $1.74 \pm 0.17$ & $1.76 \pm 0.17$ & 0.48 \\
\hline
\end{tabular}

Table 2: Angiographic findings.

\begin{tabular}{|llll|}
\hline Parameters & Cohort-I & Cohort-II & $p$-Value \\
\hline Type of Vessel & & & \\
LAD & $66.34 \%(67)$ & $54.45 \%(55)$ & 0.09 \\
RCA & $21.78 \%(22)$ & $23.76 \%(24)$ & 0.74 \\
LAD \& RCA & $4.95 \%(5)$ & $3.96 \%(4)$ & 0.73 \\
LCX & $3.96 \%(4)$ & $10.89 \%(11)$ & 0.07 \\
RAMUS & $1.98 \%(2)$ & $0 \%(0)$ & 0.29 \\
SVG Graft & $0.99 \%(1)$ & $0 \%(0)$ & 0.50 \\
OM-2 & $0 \%(0)$ & $3.96 \%(4)$ & 0.14 \\
LCX \&LAD & $0 \%(0)$ & $0.99 \%(1)$ & 0.50 \\
LMCA & $0 \%(0)$ & $0.99 \%(1)$ & 0.50 \\
RCA \& LCX & $0 \%(0)$ & $0.99 \%(1)$ & 0.50 \\
\hline Lesion Class & & & \\
A & $13.86 \%(14)$ & $14.85 \%(15)$ & 0.84 \\
B1 & $20.79 \%(21)$ & $22.77 \%(23)$ & 0.73 \\
B2 & $25.74 \%(26)$ & $25.74 \%(26)$ & 1.00 \\
C & $39.6 \%(40)$ & $36.63 \%(37)$ & 0.66 \\
\hline \% Stenosis & & & \\
>90\% & $49.5 \%(50)$ & $52.47 \%(53)$ & 0.67 \\
$80-90 \%$ & $48.51 \%(49)$ & $44.55 \%(45)$ & 0.57 \\
$<80 \%$ & $1.98 \%(2)$ & $2.97 \%(3)$ & 0.65 \\
\hline Calcification & \multicolumn{3}{|l}{} \\
None/Mild & $72.27 \%(73)$ & $70.29 \%(71)$ & 0.76 \\
Moderate & $19.8 \%(20)$ & $19.8 \%(20)$ & 1.00 \\
Severe & $7.92 \%(8)$ & $9.9 \%(10)$ & 0.62 \\
\hline Tortuosity & & & \\
$<45$ & $90.09 \%(91)$ & $82.17 \%(83)$ & 0.11 \\
$45-90$ & $9.9 \%(10)$ & $17.82 \%(18)$ & 0.11 \\
\hline Thrombus & $45.54 \%(46)$ & $36.63 \%(37)$ & 0.2 \\
\hline Pre TIMI Flow & & & \\
0 & $41.58 \%(42)$ & $52.47 \%(53)$ & 0.12 \\
1 & $34.65 \%(35)$ & $23.76 \%(24)$ & 0.09 \\
2 & $18.81 \%(19)$ & $14.85 \%(15)$ & 0.45 \\
3 & $4.95 \%(5)$ & $8.91 \%(9)$ & 0.27 \\
\hline
\end{tabular}




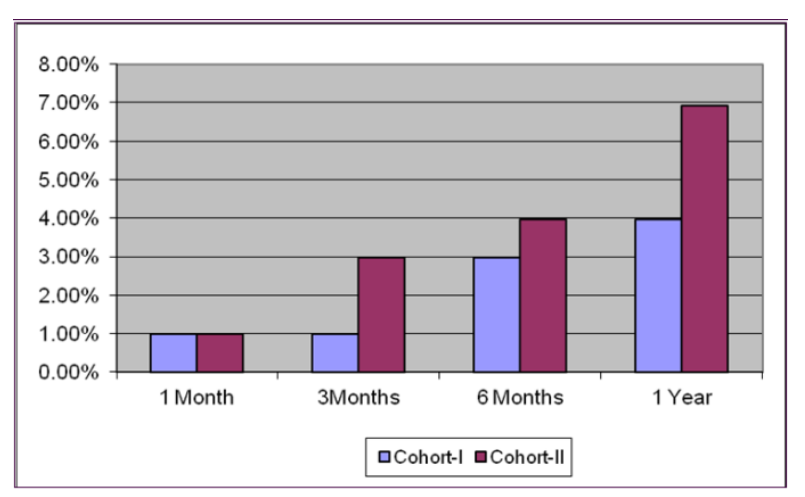

Figure 4: Over all major adverse cardiac events with duration.

\section{DISCUSSION}

Our study compared the clinical outcome of drug-eluting stents versus bare-metal stents in a large cohort patients treated with coronary stents at the private tertiary care hospital. The long-term follow-up was complete. There was a trend toward a lower event rate during the initial 6 months and a consistently higher event rate thereafter. The likelihood that these events were caused by stent thrombosis is strengthened by the demonstration of incomplete neointimal coverage as a probable reason for late stent thrombosis in patients with drug-eluting stents. Safety and efficacy study result demonstrated that over all MACE and mortality rate appears lower in DES group compare to BMS group. However, it was not statistically significant. Further long term study is required to get more viable results with larger population.

\section{ACKNOWLEDGEMENTS}

The authors thank Kakadiya Hospital, Ahmedabad, for permitting to conduct the study and collection of data.

\section{REFERENCES}

1. Michaels AD, Chatterjee K. Cardiology patient pages. Angioplasty versus bypass surgery for coronary artery disease. Circulation 2002; 106:18790.

2. Xavier D, Pais P, Xie C, et al. Treatment and outcomes of acute coronary syndromes in India (CREATE): a prospective analysis of registry data. Lancet 2008; 371:1435-42.

3. Bavry AA, Kumbhani DJ, Helton TJ, Borek PP, Mood GR, Bhatt DL. Late thrombosis of drugeluting stents: a meta-analysis of randomized clinical trials. Am J Med 2006; 119:1056-61.

4. Nordmann AJ, Briel M, Bucher HC. Mortality in randomized controlled trials comparing drug-eluting vs. bare metal stents in coronary artery disease: a meta-analysis. Eur Heart J 2006; 27:2784-814.

5. Daemen J, Wenaweser P, Tsuchida K, Abrecht L, Vaina S, Morger C, et al. Early and late coronary stent thrombosis of sirolimus-eluting and paclitaxel- eluting stents in routine clinical practice: data from a large two-institutional cohort study. Lancet 2007; 369:667-78.

6. Aoki J, Colombo A, Dudek D, et al. Peristent remodeling and neointimal suppression 2 years after polymer-based, paclitaxel-eluting stent implantation: insights from serial intravascular ultrasound analysis in the TAXUS II stud. Circulation 2005; 112:387683.

7. Fujii K, Carlier SG, Mintz GS, et al. Stent underexpansion and residual reference segment stenosis are related to stent thrombosis after sirolimus-eluting stent implantation: an intravascular ultrasound study. J Am Coll Cardiol 2005; 45:995-8.

8. Pfisterer M, Brunner-La Rocca HP, Buser PT, et al. Late clinical events after clopidogrel discontinuation may limit the benefit of drug-eluting stents: an observational study of drug-eluting versus baremetal stents. J Am Coll Cardiol 2006; 48:2584-91. 